\title{
Adjuvant chemotherapy for intrahepatic cholangiocarcinoma: far from a clinical consensus
}

\author{
Wei-Feng Qu, Wei-Ren Liu, Ying-Hong Shi \\ Department of Liver Surgery, Liver Cancer Institute, Zhongshan Hospital, Fudan University, Key Laboratory of Carcinogenesis and Cancer \\ Invasion of the Ministry of Education, Shanghai, China \\ Correspondence to: Dr. Ying-Hong Shi. Department of Liver Surgery, Liver Cancer Institute, Zhongshan Hospital, Fudan University, 180 Fenglin \\ Road, Shanghai 200032, China. Email: shi.yinghong@zs-hospital.sh.cn. \\ Comment on: Altman AM, Kizy S, Marmor S, et al. Adjuvant chemotherapy for intrahepatic cholangiocarcinoma: approaching clinical practice \\ consensus? Hepatobiliary Surg Nutr 2020;9:577-86.
}

Submitted Sep 03, 2021. Accepted for publication Oct 22, 2021.

doi: $10.21037 / \mathrm{hbsn}-21-362$

View this article at: https://dx.doi.org/10.21037/hbsn-21-362

Intrahepatic cholangiocarcinoma (iCCA), which arises from the second-order bile tracts, is the second most common primary liver cancer following hepatocellular carcinoma (1). As a subtype of biliary tract cancer (BTC), iCCA is a highly lethal neoplasm with an increasing incidence and mortality. Surgery remains the mainstay for curative intention. However, the postoperative prognosis of iCCA patients is dismal, with a 3 -year recurrence rate over $50 \%$ and a median overall survival (OS) time of approximately 30 months (2). In an attempt to improve the clinical outcome of iCCA patients, considerable efforts have been made to explore the optimal adjuvant therapy.

So far, most studies focusing on the adjuvant treatment for iCCA were retrospective and covered local to systematic therapy, including external beam radiation, transarterial chemoembolization (TACE), chemotherapy, targeted therapy, and immune therapy (3). Previous studies have suggested that patients who received adjuvant therapy were characterized by an advanced tumor stage (4), and patients with adverse prognostic factors such as high tumor burden (5) were more likely to benefit from adjuvant treatment. Notably, Miura et al. explored a significant improvement in median OS after adjuvant chemotherapy among patients with lymphoid metastasis after resection (6). However, the selection bias and insufficient treatment information in this retrospective analysis prevented its conclusions from being converted into widespread clinical utilization.

Recently, two randomized clinical trials (RCTs) focusing on adjuvant chemotherapy for BTC were published. The BILCAP study recommended an oral capecitabine regimen as the first-line adjuvant chemotherapy for BTC, which showed improved OS compared to the observation group in per-protocol analysis (53 months in the capecitabine group vs. 36 months in the observation group) (7). Controversially, the PRODIGE 12 study reported no significant difference in both recurrence-free survival (RFS) and OS between the gemcitabine plus oxaliplatin (GEMOX) arm and the surveillance arm (8). Several design flaws limited the reliability of these two clinical trials, including the long study span and the setting of blank controls. Moreover, the lack of medication information after relapse potentially overstated the effectiveness to a higher extent. Therefore, more rigorous RCTs specialized in adjuvant therapy for iCCA are urgently needed.

Altman et al. (9) have retrospectively collected robust iCCA patients between 2000 and 2014 from the National Cancer Institute's Surveillance Epidemiology and End Results (SEER) program database, and evaluated the prognostic patterns as well as the efficacy of adjuvant chemotherapy. Increasing utilization of chemotherapy was observed over the three time periods, especially in the lymph node (LN)-positive and T3/T4 disease populations. Among patients with LN-positive disease, the median OS increased from 19 to 23 months when patients accepted chemotherapy. Likewise, receipts of chemotherapy significantly decreased the hazard ratio (HR) of death in patients with $\mathrm{LN}$-positive disease $(\mathrm{HR}=0.46,95 \% \mathrm{CI}$ : 
$0.30-0.72 ; \mathrm{P}=0.001)$, as opposed to the entire cohort.

The study depicted the ascending trend for the use of adjuvant chemotherapy over the past two decades. Adjuvant chemotherapy is crucial, specifically to those with highrisk recurrence prognostic factors. Patients diagnosed with LN-positive iCCA were characterized by poor survival time and high tendency to benefit from adjuvant chemotherapy. These results were consistent with previous findings (6).

Besides the novel viewpoints presented, there were some defects in this study. Firstly, the data collection represented a large span and poor data integrity. Simple data from a retrospective database failed to explain the rapid development of adjuvant treatment strategy for iCCA in recent years. Secondly, specific description of the adjuvant chemotherapy regimen was not mentioned in the article since it was the foremost indicator for medication guidance. Thirdly, the standardization of lymphadenectomy in radical surgery was not included, which could affect the certainty of the conclusion that LN-positive patients could benefit from chemotherapy. Fourthly, the resection status and recurrence-related information was not included in the analysis, which may lead to overestimation of the chemotherapeutic effect.

Based on the current research conclusions and the design of this paper, it is difficult to reach a clinical practice consensus on decision-making for adjuvant chemotherapy. Standardization is needed in several parts of the study design. Curative liver resection, including LN skeletonization, should be detailed in future studies. A challenging viewpoint given by Zhang et al. recommended that standard lymphadenectomy of at least six LNs for iCCA possessed the greatest chance of better OS (10). Therefore, specification of the surgical procedures is a prerequisite to comparative analysis. Also, the diverse chemotherapeutic regimens for iCCA, including capecitabine, GEMOX, and paclitaxel-albumin, render the unification of adjuvant chemotherapy the top priority when selecting the study population. More importantly, reliable conclusions should be drawn by well-designed, robust RCTs.

The emergence of immune therapy is a milestone in anti-tumor treatment. Recently, phase II RCTs of monoimmunotherapy (11) or combination immunotherapy (12) proved the tolerability and efficacy of immune checkpoint inhibitors (ICIs), exemplified by programmed cell death-1 (PD-1) and cytotoxic T-lymphocyte-associated protein 4 (CTLA4) antibodies, in advanced BTCs. Considering that chemotherapy is still the basic treatment for BTCs, the regimen of ICIs plus GEMOX also exhibited promising anti-tumor activity (13).

In the era of precision treatment, targeted drugs for epidermal growth factor receptor (EGFR), vascular epidermal growth factor (VEGF), fibroblast growth factor receptor (FGFR), and isocitrate dehydrogenase 1 or 2 (IDH1/2) mutations have demonstrated a favorable response rate and improved survival in advanced cholangiocarcinoma (14). Lenvatinib, an oral multi-kinase inhibitor, has been approved for treatment of multiple solid tumors, including hepatocellular carcinoma (15). Our institution proposed an ongoing RCT of "Anti-PD1 antibody Toripalimab, Lenvatinib and GEMOX chemotherapy as first-line treatment of advanced and unresectable intrahepatic cholangiocarcinoma" in the ASCO 2020. Our primary findings were promising, with an overall response rate of $80 \%$ and three successful cases of conversion surgery in a total cohort of 30 patients. The high efficiency of combination therapy shed light on future clinical utilization.

Adjuvant therapy plays a critical role in improving survival time and preventing recurrence of iCCA. The breakthrough data of targeted immunotherapy or targeted immunotherapy combined with chemotherapy in advanced iCCA provide a novel strategy for the adjuvant treatment of resectable iCCA. Well-designed RCTs on adjuvant therapy are urgently needed for evidence-based consensus.

\section{Acknowledgments}

Funding: None.

\section{Footnote}

Provenance and Peer Review: This article was commissioned by the editorial office, Hepatobiliary Surgery and Nutrition. The article did not undergo external peer review.

Conflicts of Interest: All authors have completed the ICMJE uniform disclosure form (available at https://hbsn. amegroups.com/article/view/10.21037/hbsn-21-362/coif). The authors have no conflicts of interest to declare.

Ethical Statement: The authors are accountable for all aspects of the work in ensuring that questions related to the accuracy or integrity of any part of the work are appropriately investigated and resolved.

Open Access Statement: This is an Open Access article distributed in accordance with the Creative Commons 
Attribution-NonCommercial-NoDerivs 4.0 International License (CC BY-NC-ND 4.0), which permits the noncommercial replication and distribution of the article with the strict proviso that no changes or edits are made and the original work is properly cited (including links to both the formal publication through the relevant DOI and the license). See: https://creativecommons.org/licenses/by-nc-nd/4.0/.

\section{References}

1. Sirica AE, Gores GJ, Groopman JD, et al. Intrahepatic Cholangiocarcinoma: Continuing Challenges and Translational Advances. Hepatology 2019;69:1803-15.

2. Tian MX, Zhou YF, Qu WF, et al. Histopathologybased immunoscore predicts recurrence for intrahepatic cholangiocarcinoma after hepatectomy. Cancer Immunol Immunother 2019;68:1369-78.

3. Chun YS, Javle M. Systemic and Adjuvant Therapies for Intrahepatic Cholangiocarcinoma. Cancer Control 2017;24:1073274817729241.

4. Sur MD, In H, Sharpe SM, et al. Defining the Benefit of Adjuvant Therapy Following Resection for Intrahepatic Cholangiocarcinoma. Ann Surg Oncol 2015;22:2209-17.

5. Tsilimigras DI, Hyer JM, Paredes AZ, et al. Tumor Burden Dictates Prognosis Among Patients Undergoing Resection of Intrahepatic Cholangiocarcinoma: A Tool to Guide Post-Resection Adjuvant Chemotherapy? Ann Surg Oncol 2021;28:1970-8.

6. Miura JT, Johnston FM, Tsai S, et al. Chemotherapy for Surgically Resected Intrahepatic Cholangiocarcinoma. Ann Surg Oncol 2015;22:3716-23.

7. Primrose JN, Fox RP, Palmer DH, et al. Capecitabine compared with observation in resected biliary tract cancer (BILCAP): a randomised, controlled, multicentre, phase 3 study. Lancet Oncol 2019;20:663-73.

Cite this article as: Qu WF, Liu WR, Shi YH. Adjuvant chemotherapy for intrahepatic cholangiocarcinoma: far from a clinical consensus. HepatoBiliary Surg Nutr 2021;10(6):887889. doi: 10.21037/hbsn-21-362
8. Edeline J, Benabdelghani M, Bertaut A, et al. Gemcitabine and Oxaliplatin Chemotherapy or Surveillance in Resected Biliary Tract Cancer (PRODIGE 12-ACCORD 18-UNICANCER GI): A Randomized Phase III Study. J Clin Oncol 2019;37:658-67.

9. Altman AM, Kizy S, Marmor S, et al. Adjuvant chemotherapy for intrahepatic cholangiocarcinoma: approaching clinical practice consensus? Hepatobiliary Surg Nutr 2020;9:577-86.

10. Zhang XF, Xue F, Dong DH, et al. Number and Station of Lymph Node Metastasis After Curative-intent Resection of Intrahepatic Cholangiocarcinoma Impact Prognosis. Ann Surg 2021;274:e1187-95.

11. Kim RD, Chung V, Alese OB, et al. A Phase 2 Multiinstitutional Study of Nivolumab for Patients With Advanced Refractory Biliary Tract Cancer. JAMA Oncol 2020;6:888-94.

12. Klein O, Kee D, Nagrial A, et al. Evaluation of Combination Nivolumab and Ipilimumab Immunotherapy in Patients With Advanced Biliary Tract Cancers: Subgroup Analysis of a Phase 2 Nonrandomized Clinical Trial. JAMA Oncol 2020;6:1405-9.

13. Chen $\mathrm{X}, \mathrm{Wu} \mathrm{X}, \mathrm{Wu} \mathrm{H}$, et al. Camrelizumab plus gemcitabine and oxaliplatin (GEMOX) in patients with advanced biliary tract cancer: a single-arm, open-label, phase II trial. J Immunother Cancer 2020;8:e01240.

14. Kelley RK, Bridgewater J, Gores GJ, et al. Systemic therapies for intrahepatic cholangiocarcinoma. J Hepatol 2020;72:353-63.

15. Kudo M, Finn RS, Qin S, et al. Lenvatinib versus sorafenib in first-line treatment of patients with unresectable hepatocellular carcinoma: a randomised phase 3 noninferiority trial. Lancet 2018;391:1163-73.

(English Language Editor: A. Kassem) 